\title{
Development and Implementation of a Low-Cost Switching Control Software for Multi-level Converter
}

\author{
Emilienne Leugoue $^{1 *}$, Paul Gistain Ipoum-Ngome ${ }^{2}$, Zhang Jianhua ${ }^{1}$, Daniel L. Mon-Nzongo ${ }^{2}$ \\ ${ }^{1}$ North china Electric Power University, No.2 Beinong Road, Changping District, 100622, Beijing, China. \\ 2 Institute of Fuzhou University, Fuzhou, China. \\ * Corresponding author. Tel.: +8615652684721; email: emiliejolie90@yahoo.fr \\ Manuscript submitted April 10, 2019; accepted June 21, 2019. \\ doi: 10.17706/ijcee.2019.11.3.143-154
}

\begin{abstract}
Multilevel converters have been designed and implemented many decades ago in many countries around the word. However, in West Africa, these systems are difficult to implement because of lack of expertise and hardware which are usually very expensive. This paper proposes an implementation of an Embedded Switching Control Software (ESCS) code on Arduino 2560 hardware for multi-level systems using Model-Based Design (MBD) approach that Arduino 2560 control hardware platforms are low-cost system and locally available in many Africa countries. Thus, it can enable the validation of complex energy conversion systems using local components for research and education purposes. The paper provides detailed steps to reach such goals by proposing a Carrier Based Space Vector Pulse Wide Modulation (CBSVPWM) for three-phase multi-level inverters. A simple model of CB-SVPWM is considered to automatically generate the ESCS code for a single-phase five-level Cascaded H-Bridge (CHB) inverter then deployed on Arduino 2560 hardware conforming to On-Target Rapid Prototyping (OTRP) approach. The simulated and practical results confirm that MBD with Simulink offers a convenient approach to develop ESCS code for multi-level converters.
\end{abstract}

Key words: Development, implementation, embedded switching control software, multi-level converter.

\section{Introduction}

Nowadays, multi-level converter technology is playing an increasingly important role in power conditioning systems such as High Voltage DC transmission (HVDC) and Distributed Energy Resource (DER) systems [1]. The multi-level converters are power electronic circuits that can provide output voltage with more than two levels and be operated in inverter or rectifier mode [1], [2]. Multi-level converters are generally controlled by Space Vector PWM (SVPWM) or Carrier-Based Space Vector PWM (CB-SVPWM) [3]. SVPWM and CB-SVPWM called switching control scheme in this paper are actually implemented digitally. Digital implementation of switching control scheme is an Embedded Switching Control Software (ESCS) code deployed on an Embedded Control Hardware (ECH) by using Manual Programming (MP) or ModelBased Design (MBD) approach. MP method is the development of ESCS code by using Hand-programming and Integrated Development Environment (IDE) tools. However, due to the tedious error-prone handprogramming procedures, MBD has become more attractive than MP approach. Point of view of new designers, MBD is a possible procedure for making them more focus on control system design, implementation and evaluation rather than on time-consuming low-level programming [4]. 
MBD has been a discussion topic for decades but has only in recent years evolved into a complete design flow from model creation to implementation. In literature several works were investigated to develop ESCS code for power electronics applications. The ref. [5] described a Rapid Control Prototyping (RCP) using MATLAB/Simulink-RTW and DSP-FPGA based motor controller. It developed an ECH named DSP-2 constituted of Texas Instruments (TI) TMS320C32 and Xilinx XCS40PQ240 devices. The developed DSP-2 enables graphical programming using DSP-2 library from Simulink and automatic code generation conforming to RCP approach. However, that DSP-2 hardware and software were developed is not available for commercial purpose. Mathworks Inc. has released a complete workflow of MBD by introducing the concept On-target Rapid prototyping (OTRP) [6]. OTRP is a method that allows designers to automatically generate a production Code from a Simulink model and provides possibilities to generate production code that can be directly downloaded to the embedded fixed or floating-point ECH compatible with MATLAB/Simulink. The ref. [7] described the detailed steps in building a MBD platform around an Advanced RISC Machine (ARM) based embedded three-phase motor control. This work proposed by Analog Devices' engineers shows out the new commercial hardware and software tools for AC motor control fed by two level converter. But the cost of this platform is an issue for students or researchers without enough financial supports. The ref. [8] proposed an ESCS code for five-level inverter using Xilinx EC3S000E FPGA platform and MATLAB/Simulink /Xilinx System Generator (XGS). However the developed code requires XGS toolbox library installed to Simulink dedicated to Xilinx products. Therefore, the generated code is not reusable for FPGAs provided by other suppliers.

In order to mitigate the drawbacks listed above, this paper has proposed a first ever implementation of ESCS code on Arduino 2560 platform for multi-level converter by using MBD approach with MATLAB/Simulink. The generated code from Simulink model can be reusable for all ECH compatible with MATLAB/Simulink based OTRP approach. Because of availability and the lower cost of Arduino 2560 hardware the main purpose of this work is to provide students and new designers a way to rapidly implement ESCS for high power converters using Simulink tools.

The content of this paper is subdivided into three sections. The first section explains MBD workflow with MATLAB/Simulink. The second section develops mathematical model of CB-SVPWM and explains OTRP workflow. A single phase CB-SVPWM for five-level inverter was considered as case of study. The last section describes the practical test-bench platform developed in laboratory and presents also the phase of deployment and verification of the ESCS code running on Arduino hardware.

\section{Model-Based Design in MATLAB/Simulink}

MBD refers to the use of models and modelling as the basis for embedded control software (ECS) development. The traditional approach to design ECS software is through MP in a computer language of the target hardware. MP process has become less attractive because of some limitations such as human errors and time-consuming. Fig. 1 shows the block diagram describing the MBD process. From this figure, system model is the process of modelling, simulation of physical plant and the designing of the embedded controller using MATLAB code and Simulink blocks. Generate code is the process of automatic code generation from Simulink model after modelling and simulation. The embedded Hardware embedded the ECS code. ECS code is constituted of control algorithm and ESCS code or PWM software code which generates the pulse waveforms to effectively monitor the physical plant through power converter. The physical plant is a dynamical system that can be modelled and implemented in full or scaled-down model in real-world or numerically implemented on the real time target machine. MBD workflow can be resumed as activities of modelling, simulation, rapid prototyping; embedded deployment (implementation), testing and verification. 


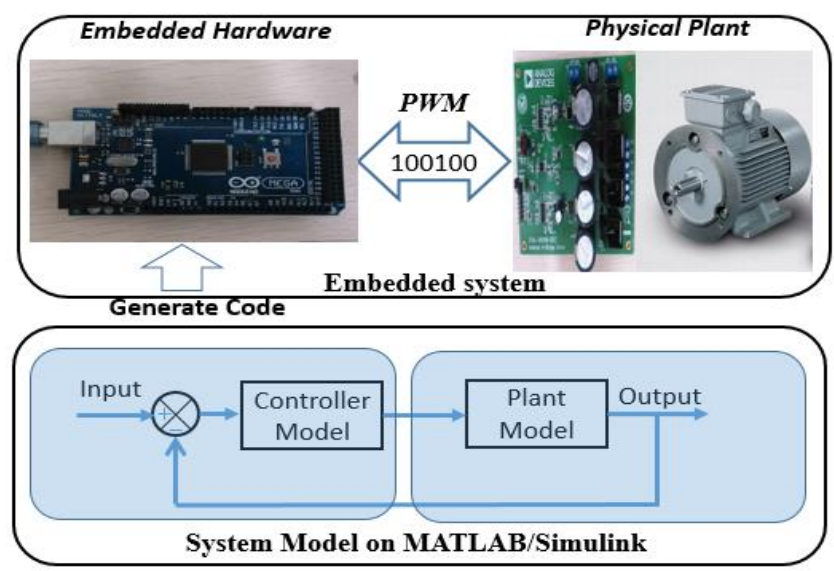

Fig. 1. Block diagram of model-based design approach.

Fig. 2 shows block diagram of MBD workflow. The following paragraph describes the steps of MBD workflow.

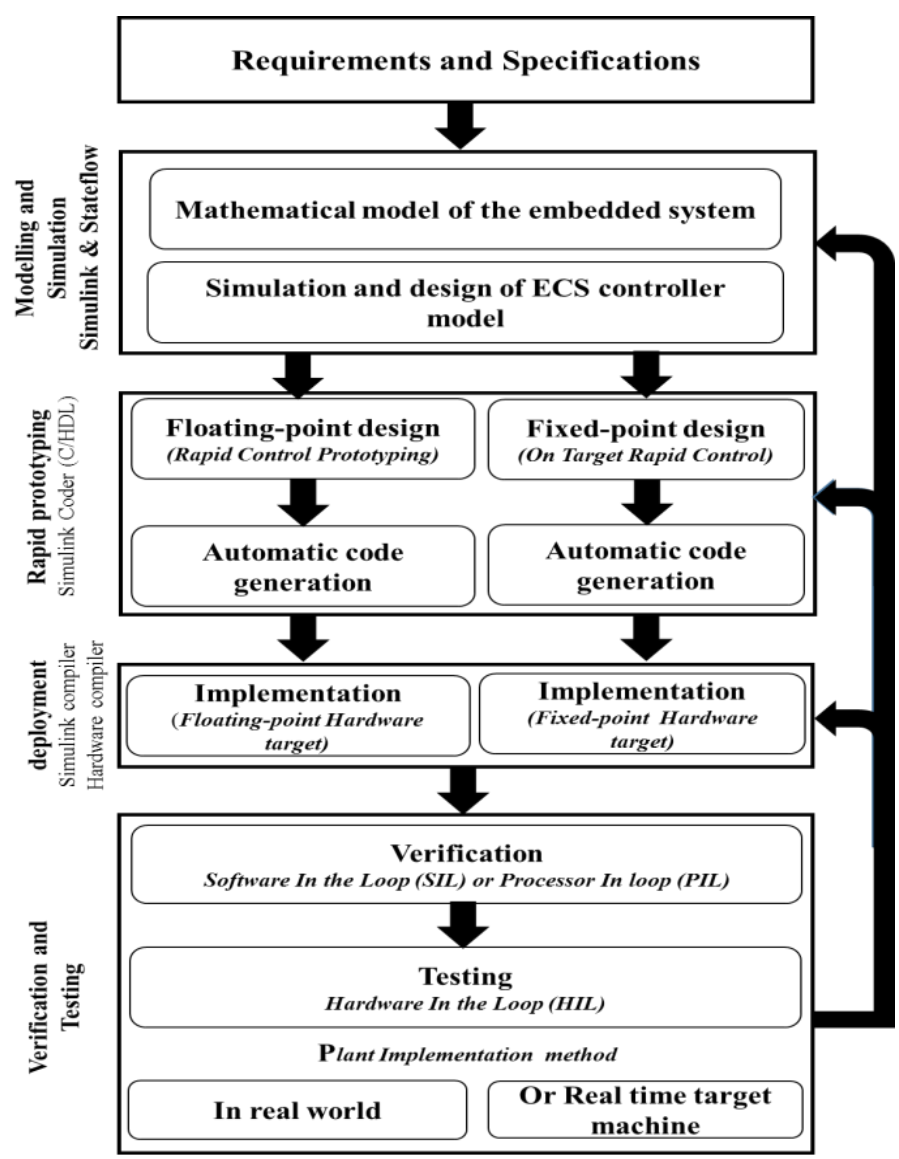

Fig. 2. Model based design workflow in MATLAB/simulink.

At the first stage of the any design it is important to get requirements and specifications of the investigated system. For example, control scheme topologies and modulation strategies. After getting that the designer starts mathematical modelling and simulation of the controller and plant. Then retains only the model of the controller for the rapid prototyping process. The rapid prototyping is the development of the ECS code through rapid control prototyping (RCP) or on-target rapid prototyping (OTRP). Afterward, 
the implementation can be achieved depending on the target hardware. Simulink coder and/or IDE tools are used to generate and deploy the executable code from the embedded control model on the target hardware. Software in the loop (SIL) or Processor in the loop (PIL) verification can then started to be performed after successfully implementing the ECS code on the hardware. PIL is the comparison between the ECS model running in the computer through MATLAB/Simulink against ECS model running on the target hardware. Finally, the successful results of previous stage directly leads to the hardware in the loop testing (HIL). The HIL is the last step of MBD workflow. It is the verification and testing of the embedded system (controller and plant model) in real-time in order to verify and validate design requirements. In the HIL process, the ECS code runs normally on the embedded hardware when the plant can be implemented whether around a scaled-down model in real-world (physical implementation) or in the real-time target machine (numerical implementation).

\section{MATLAB/Simulink Model of Multi-level Converter Controlled by CB-SVPWM}

The basic block diagram of high power electronics systems is represented in Fig. 3. The plant model is constituted of multi-level converter processing the power flow between the power supply and the load. The controller model includes PI or state space regulator and PWM modulator. As multi-level converter, Neutral point clamped (NPC) and cascaded H-bridge converters (CHB) are the most popular topologies used in industry. The model of these converter can be build up using SimPowerSystems toolbox of MATLAB/ Simulink. Thus, in this paper SimPowerSystems power electronic library is then used to implement the model of the multi-level converter.

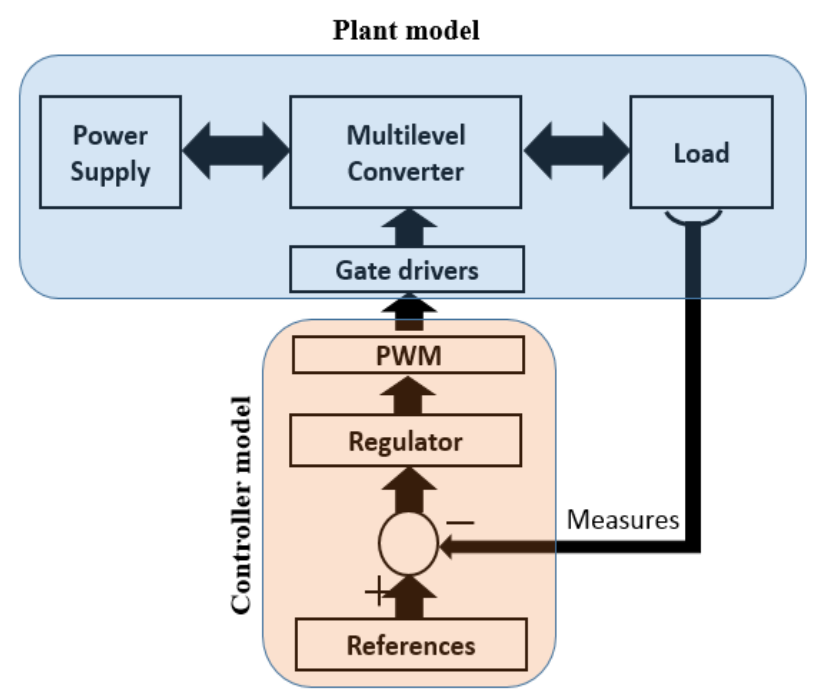

Fig. 3. Block diagram of embedded system for high power application.

\subsection{Five-Level Cascaded H-Bridge Converter}

Fig. 4 shows the representation of a single phase five-level CHB inverter. The five-level CHB inverter is build up around two H-bridges connected in serial. Each H-bridge can provide three voltage levels output and can operates whether as inverter or rectifier. The converter is supplied by two isolated DC voltages $\left(V_{d c 1}\right.$ and $\left.V_{d c 2}\right)$. In this case, the five-level converter is configured as inverter so the output phase voltage (van) is alternative and equal to the sum of partial voltages $\left(v_{a n}=v_{\text {cell1 }}+v_{\text {cell2 }}\right)$ as shown in Table 1. Table 1 represents switching states of five-level inverter where Vcell1 and Vcell2 are respectively a partial voltage of H-bridge 1 and 2. 


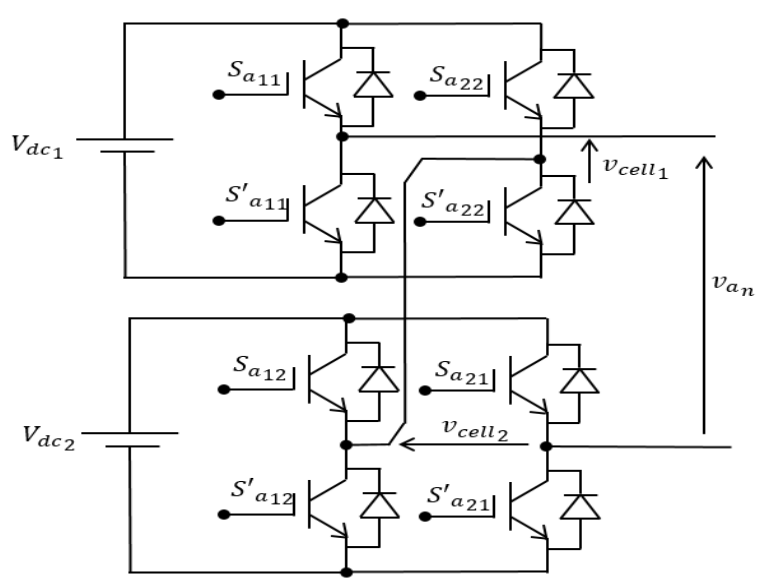

Fig. 4. Single phase 5-level CHB inverter.

Table 1. Switching States and Output Voltages in a 5-Level CHB Inverter

\begin{tabular}{ccccccc}
\hline $\mathbf{S}_{\mathrm{a} 11}$ & $\mathbf{S}_{\mathrm{a} 22}$ & $\mathbf{S}_{\mathrm{a} 12}$ & $\mathbf{S}_{\mathrm{a} 21}$ & $\mathbf{v}_{\text {cell1 }}$ & $\mathbf{v}_{\mathbf{c e l l}}$ & $\mathbf{v a}$ \\
\hline $\mathbf{1}$ & 0 & 1 & 0 & $\mathrm{~V}_{\mathrm{dc} 1}$ & $\mathrm{~V}_{\mathrm{dc} 2}$ & $\mathrm{~V}_{\mathrm{dc} 1}+\mathrm{V}_{\mathrm{dc} 2}$ \\
$\mathbf{1}$ & 0 & 1 & 1 & $\mathrm{~V}_{\mathrm{dc} 1}$ & 0 & $\mathrm{Vdc} 1$ \\
$\mathbf{1}$ & 0 & 0 & 0 & $\mathrm{~V}_{\mathrm{dc} 1}$ & 0 & $\mathrm{~V}_{\mathrm{dc} 1}$ \\
$\mathbf{1}$ & 1 & 1 & 0 & 0 & $\mathrm{~V}_{\mathrm{dc} 2}$ & $\mathrm{~V}_{\mathrm{dc} 2}$ \\
$\mathbf{0}$ & 0 & 1 & 0 & 0 & $\mathrm{~V}_{\mathrm{dc} 2}$ & $\mathrm{~V}_{\mathrm{dc} 2}$ \\
$\mathbf{0}$ & 0 & 0 & 0 & 0 & 0 & 0 \\
$\mathbf{0}$ & 0 & 1 & 1 & 0 & 0 & 0 \\
$\mathbf{1}$ & 1 & 0 & 0 & 0 & 0 & 0 \\
$\mathbf{1}$ & 1 & 1 & 1 & 0 & 0 & 0 \\
$\mathbf{1}$ & 0 & 0 & 1 & $\mathrm{~V}_{\mathrm{dc} 1}$ & $-\mathrm{V}_{\mathrm{dc} 2}$ & $\mathrm{~V}_{\mathrm{dc} 1}-\mathrm{V}_{\mathrm{dc} 2}$ \\
$\mathbf{0}$ & 1 & 1 & 0 & $-\mathrm{V}_{\mathrm{dc} 1}$ & $\mathrm{~V}_{\mathrm{dc} 2}$ & 0 \\
$\mathbf{0}$ & 1 & 1 & 1 & $-\mathrm{V}_{\mathrm{dc} 1}$ & 0 & $\mathrm{~V}_{\mathrm{dc} 2}-\mathrm{V}_{\mathrm{dc} 1}$ \\
$\mathbf{0}$ & 1 & 0 & 0 & $-\mathrm{V}_{\mathrm{dc} 1}$ & 0 & $-\mathrm{V}_{\mathrm{dc} 1}$ \\
$\mathbf{1}$ & 1 & 0 & 1 & 0 & $-\mathrm{V}_{\mathrm{dc} 2}$ & $-\mathrm{V}_{\mathrm{dc} 2}$ \\
$\mathbf{0}$ & 0 & 0 & 1 & 0 & $-\mathrm{V}_{\mathrm{dc} 2}$ & $-\mathrm{V}_{\mathrm{dc} 2}$ \\
$\mathbf{0}$ & 1 & 0 & 1 & $-\mathrm{V}_{\mathrm{dc} 1}$ & $-\mathrm{V}_{\mathrm{dc} 2}$ & $-\mathrm{V}_{\mathrm{dc} 1}-\mathrm{V}_{\mathrm{dc} 2}$ \\
\hline
\end{tabular}

\subsection{Mathematical Model of Carrier-Based SVPWM for Multilevel Converter}

Space Vector Carrier Based PWM (CB-SVPWM) is an equivalency of SVM. Fig. 5 shows a block diagram of CB-SVPWM. The CB-SVPWM for multi-level converter is an extension of carrier based PWM of 2-level converter. The principle of a classical PWM is to generate single carrier waveform and reference signals in order to compute the switching times while multi-carriers are needed for multi-level converter. The multicarriers are obtained by vertically or horizontally shifting a single carrier waveform. $\mathrm{N}_{\text {level }}-1$ carriers are then generated and compared at each time to a set of three reference voltages $m_{a}^{*}, m_{b}^{*}, m_{c}^{*}$ coming from the control strategy and added to zero sequence component $V_{z}$. The results of comparison provides a first level switching signals or switching patterns. These switching signals are reused to generate a modulated output signals according to the desired level number of the converter. Decode1 is a global decode function which decode a first level switching signal of each partial cell in each phase. Decode 2 is a local decode function used to provide switching patterns of each power device conforming to each partial cell. The optimization function is used to ensure the number of commutation balanced between partial cells. The death time function is used because of storage effect due to power switches (IGBTs or MOSFETs). The modulation index 
$M$, and the frequency index $M_{f}$ are defined by equations (1) and (2). The zero-sequence component is defined by the equation (3).

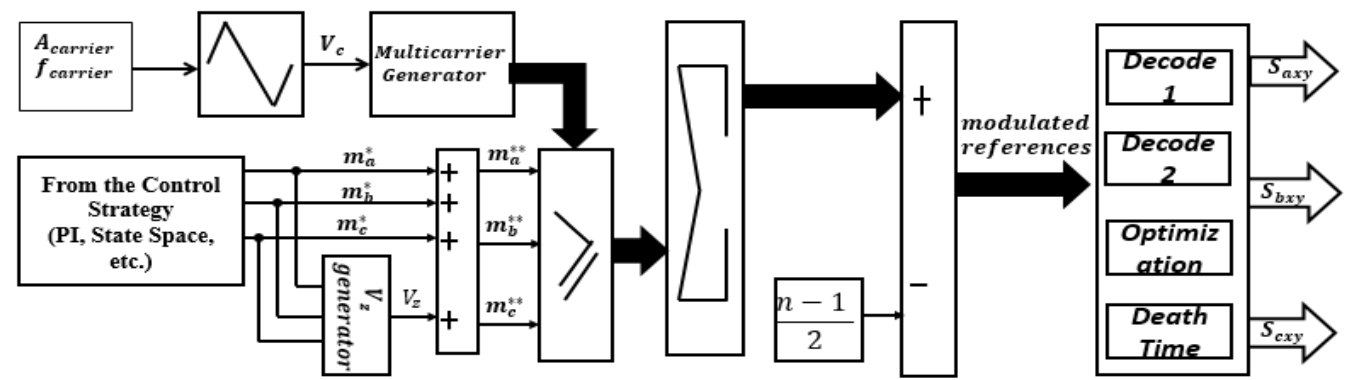

Fig. 5. Block diagram of space vector carrier based PWM for three phase multi-level converter.

$$
\begin{gathered}
M=\frac{A_{m}^{*}}{A_{c}} \\
M_{f}=\frac{f_{c}}{f_{c m}} \\
V_{z}=\frac{\left[\max \left(m_{a}^{*}+m_{b}^{*}+m_{c}^{*}\right)+\min \left(m_{a}^{*}+m_{b}^{*}+m_{c}^{*}\right)\right]}{2}
\end{gathered}
$$

with

$$
m_{a}^{* * *}=m_{a}^{*}-v_{z}, m_{b}^{* * *}=m_{b}^{*}-v_{z}, m_{c}^{* * *}=m_{c}^{*}-v_{z}
$$

And, $A_{c}$ and $f_{c}$ are respectively the amplitude and frequency of carrier signal. $A_{m}^{*}, f_{c m}$ are respectively the amplitude and frequency of the reference voltage.

\subsection{Simulation of CB-SVPWM for Multi-level Converter}
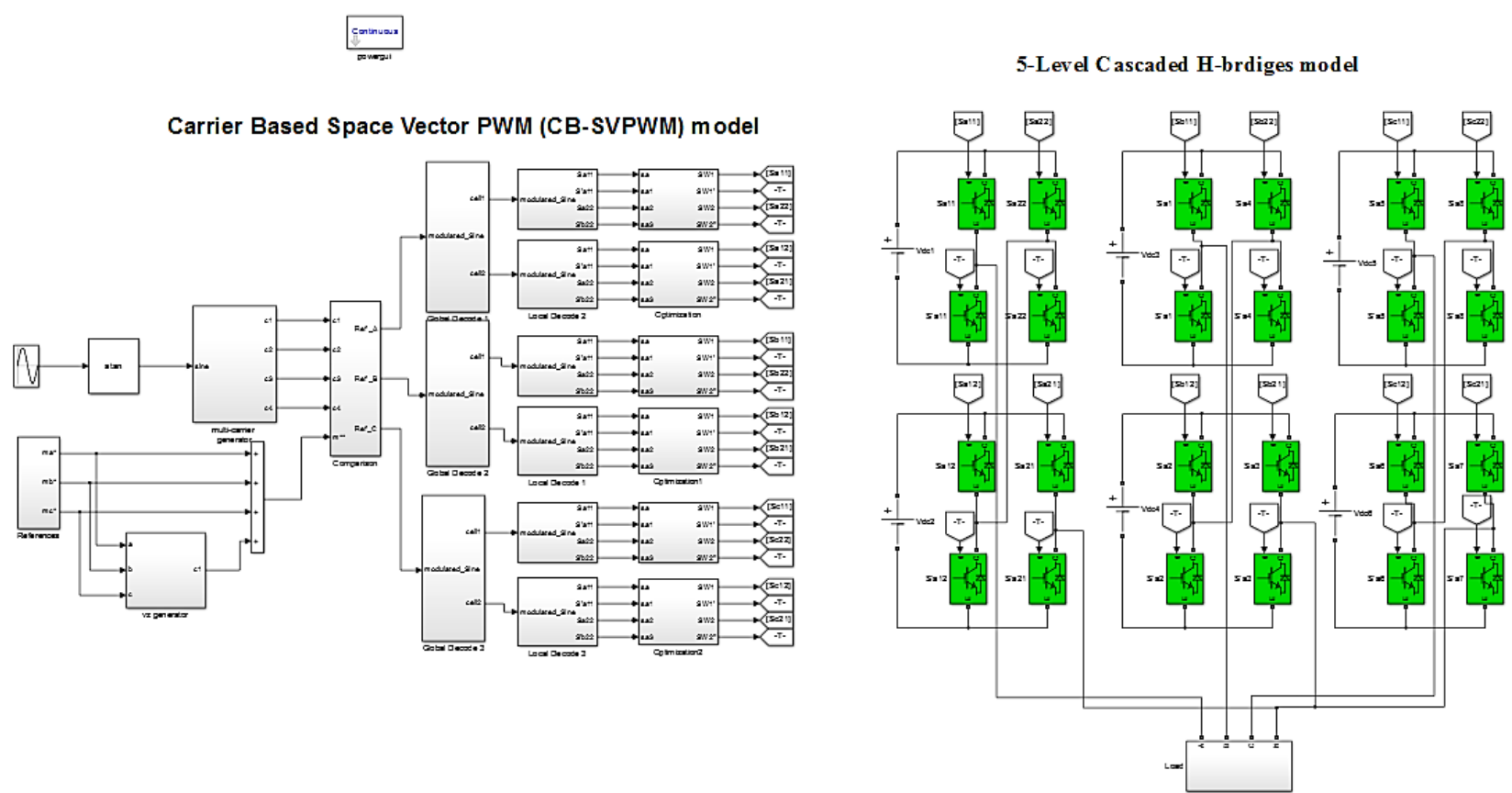

Fig. 6. MATLAB/Simulink model of 5-Level CHB inverter controlled by CB-SVPWM. 
The model of five-level three-phase CHB inverter and CB-SVPWM is implemented through MATLAB/Simulink as shown in Fig. 6. The model of five-level CHB inverter is implemented using SimPowerSystems block-sets. CB-SVPWM model is implemented as it is shown in Fig. 5. The model is first used for simulation and then readapted to automatically generate ESCS code. Fig. 7 shows the partial voltages; phase voltage and line to line voltage of a five-level inverter simulation results in continuous time model.
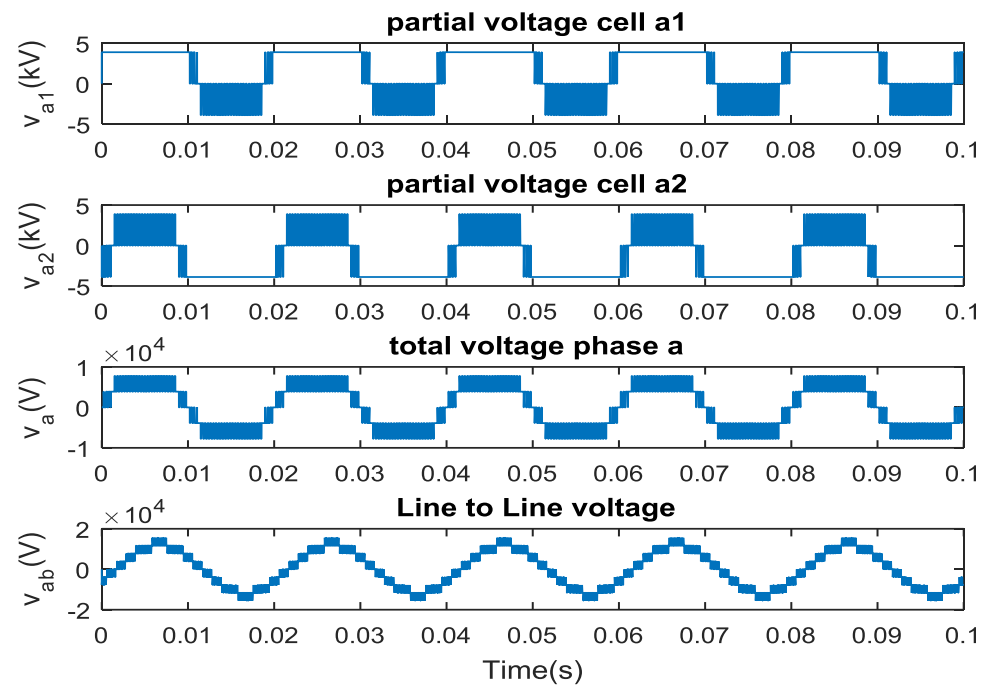

Fig. 7. Partial voltages, phase a voltage and line to line voltage of 5-level CHB inverter.

\subsection{OTRP of Embedded CB-SVPWM Software for Multi-level Converter}

The rapid prototyping and deployment of MBD workflow are steps needed to automatically generate and deploy the ECS software after modelling and simulation. Generally the model initially implemented from Simulink cannot be used to generate the ECS code .It is because of incompatibility of certain Simulink blocks with Simulink coder. RCP or OTRP approach is whether used to automatically generate ECS software code or an executable from the control model to a rapid implementation on embedded hardware platforms. The code generated can also be exported to another IDE software tools for hardware implementation. While the executable is directly deployed to a specific hardware platform without moving to another environment. But the hardware drivers are required to be installed to MATLAB/Simulink software.

Five steps are needful for developing ECS code using OTRP [6]. These steps are: 1) establishing of the model guidelines using Simulink Model Advisor, 2) establishing of code generation settings using Code Generation Advisor, 3) creation of the floating point algorithm design using modelling tools of Simulink and Stateflow, 4) conversion to fixed-point data if required using fixed-point automation tools, 5) comparison between fixed and floating-point results. The ECS software code of CB-SVPWM called in this article ESCS code was generated from steps listed above. The simulated CB-SVPWM model presented in Fig. 5 was modified conforming to Simulink Model and Code Generation Advisor. Therefore we obtained a discrete CBSVPWM model equivalent to regular sampled PWM. Thus, the model was changed from continuous time model to discrete time model by modifying all continuous signal by their digital equivalency model. Furthermore all functions (decode1, decode2, optimization) previously edited using M-file was embedded to the Simulink model using MATLAB embedded function supported by Simulink coder. The performance of this model depends on resources available on the embedded target hardware. 


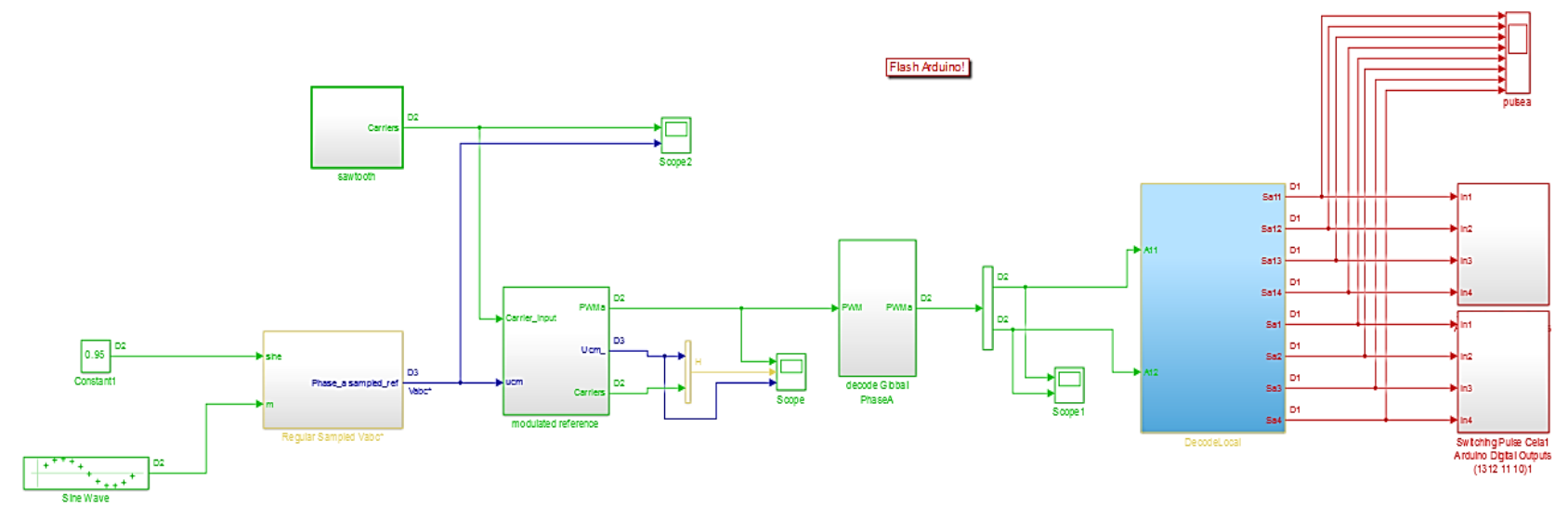

Fig. 8. Discrete model of single phase CB-SVPWM adapted to Arduino 2560 based-platform.

Fig. 8 shows the digital model of the single phase CB-SVPWM for five-level inverter adapted to Arduino 2560 platform. All Arduino drivers was previously linked to Simulink in order to deploy directly the generated ESCS code from the Simulink model to Arduino hardware. These drivers are available at Mathworks website. Fig. 9 shows a captured image of a code generation report. It contents subsystem report, code interface report, traceability report, static code metric reports and code replacement report. This report interface enables links to access to the automatic codes generated from the discrete model shown in Fig. 8. And it offers traceability of the code from the model to the code file.

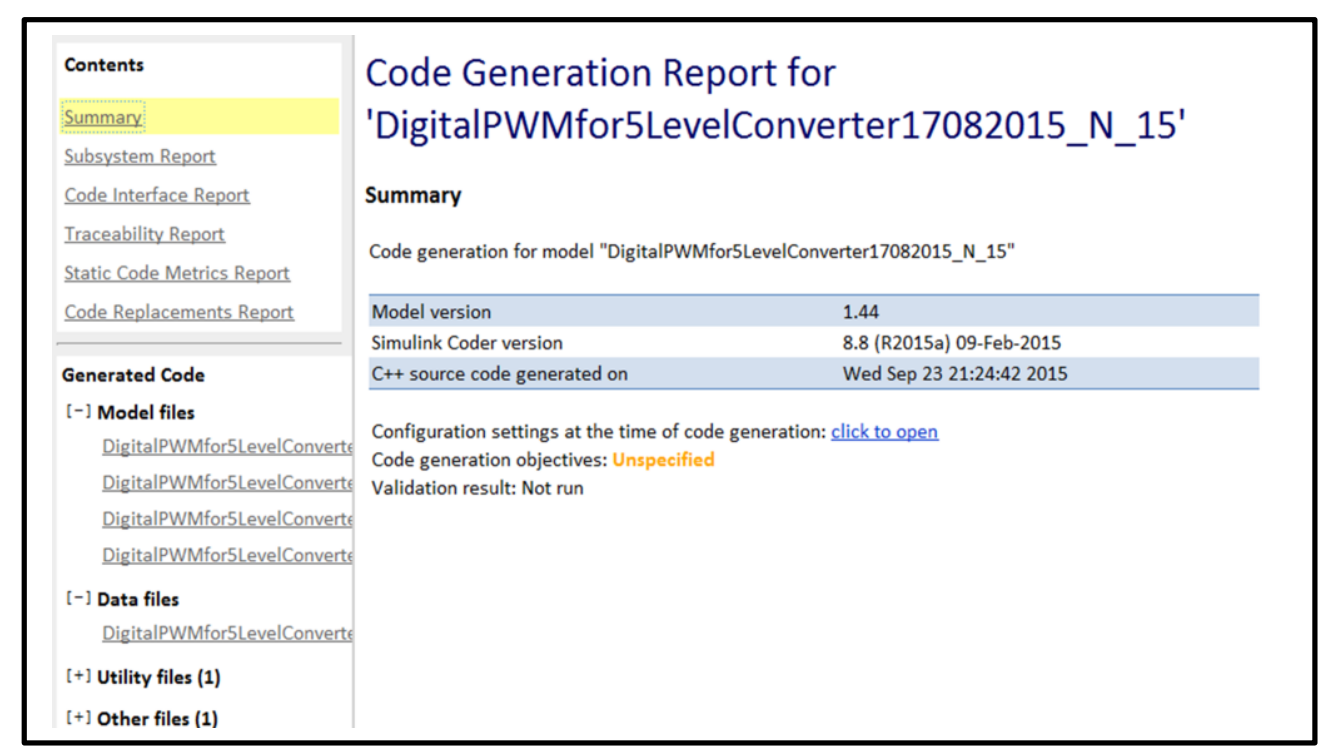

Fig. 9. Code generation report from simulink for single phase digital CB-SVPWM.

\section{Experimentation}

Fig. 10 shows the experiment test-bench developed in laboratory. It contents DC-link supplies (1), providing DC voltage to each $\mathrm{H}$-bridge cell. It is obtained through a $50 \mathrm{VA}, 380 / 36 \mathrm{~V}$ transformers and $\mathrm{AC} / \mathrm{DC}$ rectifiers. Gate driver supplies (2), it provides isolated supplies to the gate drivers. The CN0196 Hbridge of Analog Devices contents two isolated gate drivers ADmu7234. The aDmu7234 in the power side requires $12 \mathrm{~V}$ to supply the low and high side gates of each H-bridge. The Low side gate voltage is obtained 
through a 12/1A power supplies while the high side gates are supplied through the bootstrap circuits. Fivelevel converter (3), it is configured to DC/AC inverter which contents 2 series connected H-bridge CN0196 of Analog Devices. The CN0196 is constituted of FDP5800 mosfets and ADuM7234 gate drivers. The CN0196 has been modified as a three-level inverter instead of two-level as it was implemented [9]. Arduino Mega 2560 hardware (4) is an 8 bits, $16 \mathrm{MHz}$, microcontroller with $256 \mathrm{~kb}$ as flash memory with more than 50 digital inputs and outputs. Fig. 11 shows the configuration of the system during the development and deployment of a single phase five-level ESCS. During the development of ESCS code of CB-SVPWM, the Arduino Mega 2560 hardware was connected to the PC via USB port and the oscilloscope through probes as shown in Fig. 11. During the implementation, Simulink solver was configured to discrete mode with no continuous states. The step time was selected to $T_{\text {step }}=0.05 \mathrm{~ms}$ and the frequency was fixed to $f_{\text {step }}=20 \mathrm{kHz}$. The carrier frequency was chosen to be 25 time larger than the frequency of modulating signal so the frequency index $M_{f}=25$ and the modulating index was chosen to $M=0.95$.

(1) : Dc-link supplies of $\mathrm{H}-$ bridges $(\mathrm{Vdc} 1=$ $36 \mathrm{~V}, 50 \mathrm{~W}$ $\mathrm{Vdc} 2=36 \mathrm{~V}, 50 \mathrm{~W}$ )

(2) : Gate driver supplies $(12 \mathrm{~V}$ for each H-bridge)

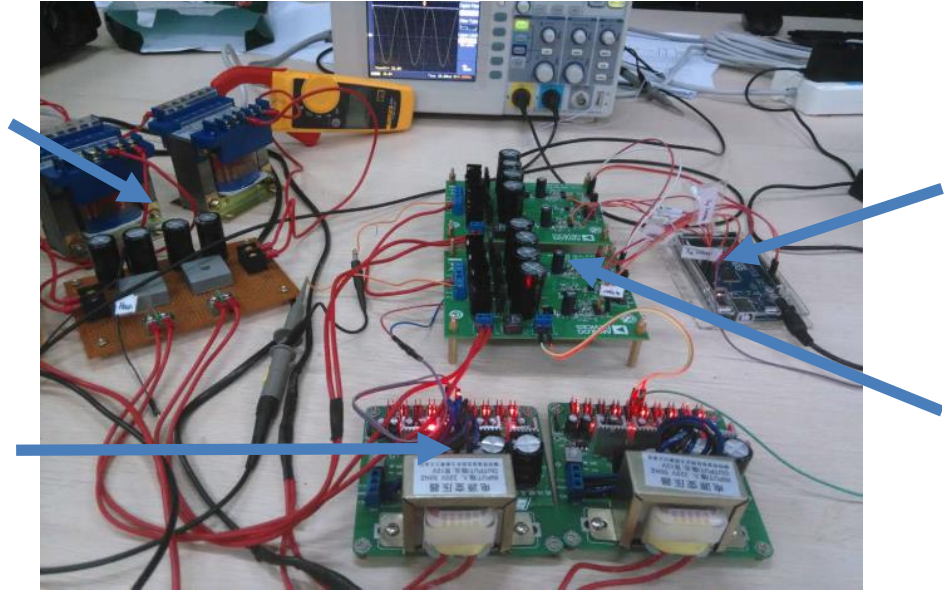

Fig. 10. Experimental platform of single phase 5-level DC/AC converter.
(4): Arduino Mega

2560

Based-platform

(3) 5-level converter: $2 *$ H-bridges AD CN0196 with isolated gate drivers Admu7234

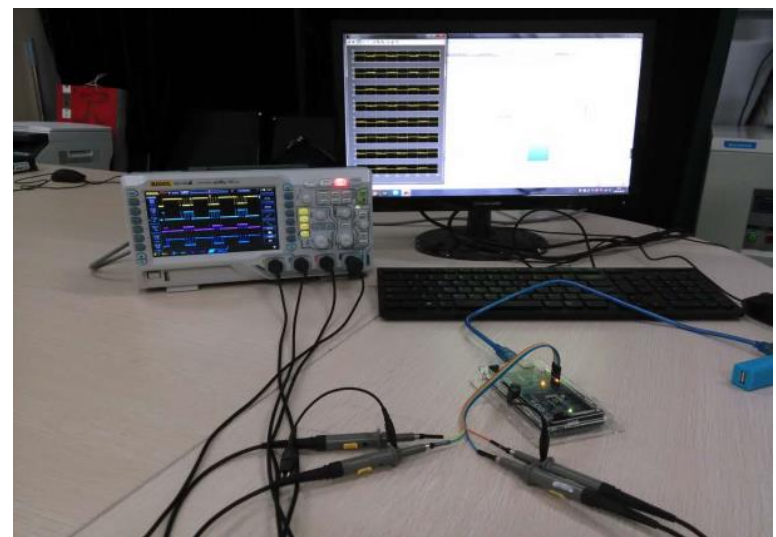

Fig. 11. Development, deployment and verification of digital CB-SVPWM software.

Fig. 12 shows the results of switching signals for each partial cell of 5-level inverter. Fig. 12a shows the results of switching pulses obtained from Simulink for cell a1 and a2. $S_{a 11}$ and $S_{a 22}$ are high side switching pulses of cell-a1, while their complementary are $\left(! S_{a 11}, ! S_{a 22}\right)$ and represents the low side switching pulses of cell-a1. In the same manner on the Fig. 12c, $S_{a 12}, S_{a 21}$ and (! $\left.S_{a 12}, ! S_{a 21}\right)$ are respectively the high and low side switching pulses of cell-a2 obtained from Simulink model. Fig. 12b and Fig. 12.d show practical results of switching pulses obtained by oscilloscope after deploying ESCS code of CB-SVPWM on Arduino 2560 
hardware. Fig. $12 \mathrm{~b}$ shows four switching pulses of cell-a1 while Fig. $12 \mathrm{~d}$ shows the switching pulses of cella2. As it shown in Fig. 12, simulated results and practical results are quite similar.

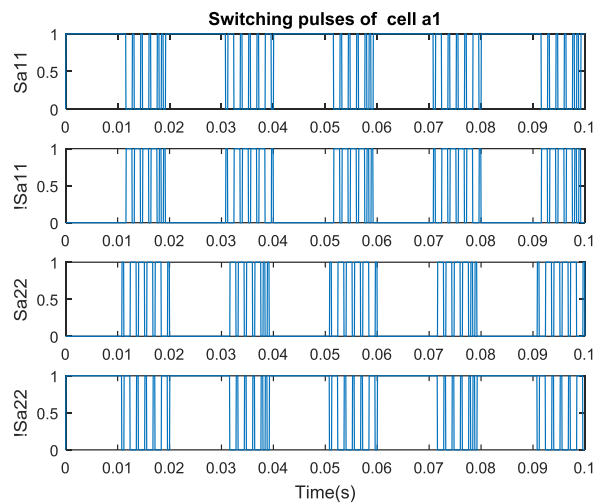

(a)

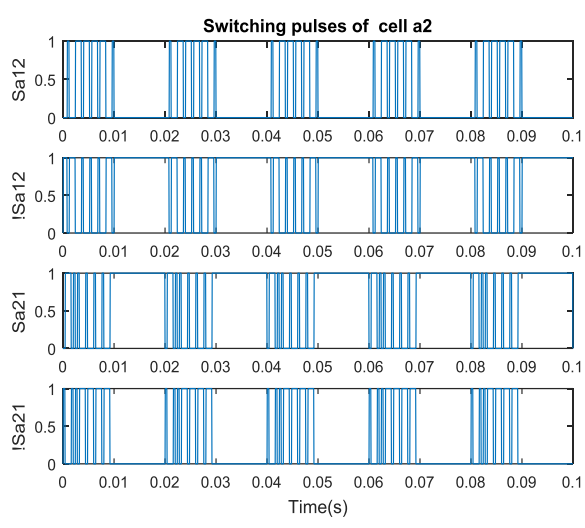

(c)

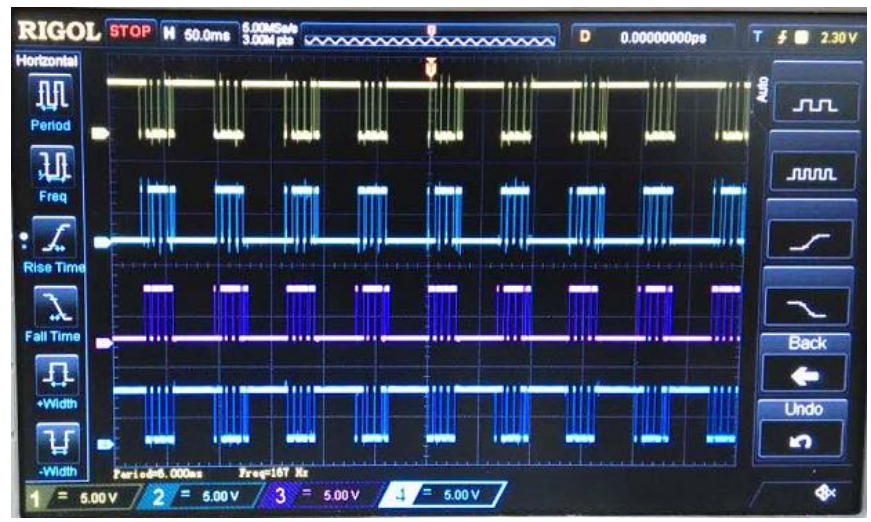

(b)

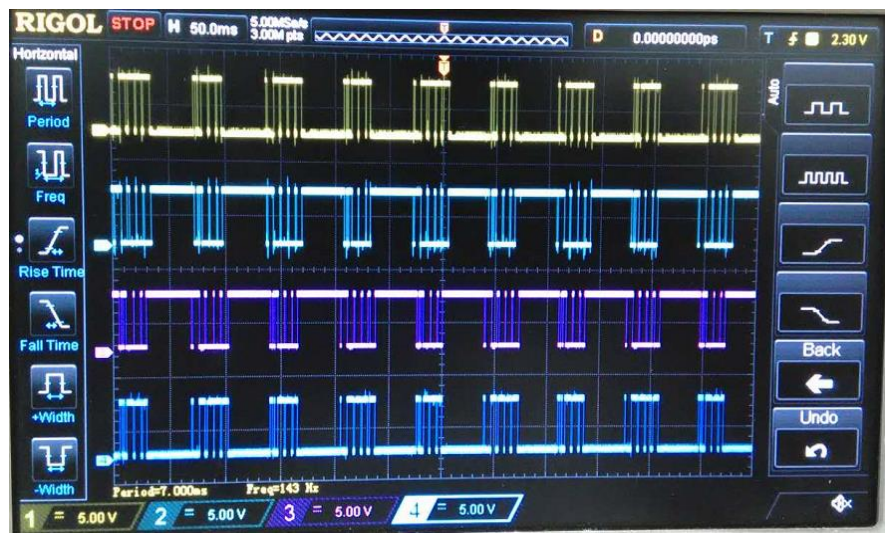

(d)

Fig. 12. Switching patterns of Digital CB-SVPWM from Simulink scopes and from oscilloscope. (a), (b): Switching control waveform of cell-a1 and cell-a2 from Simulink; (c),(d): Switching control waveform of cell-a1 and cell a2 from the oscilloscope through Arduino pins.

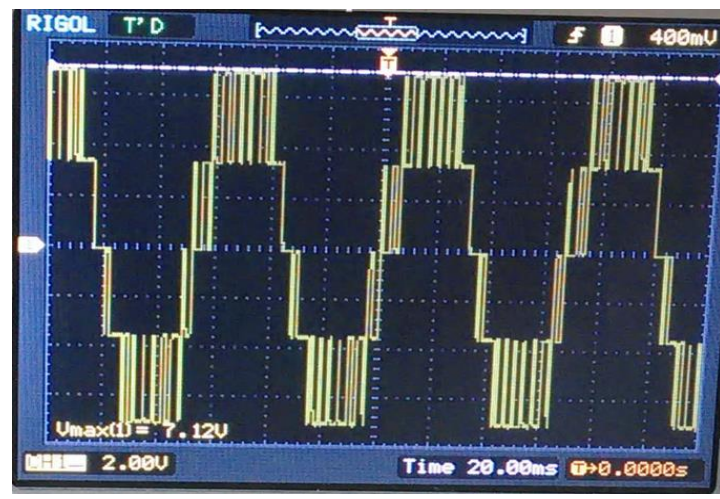

(a) van before filter

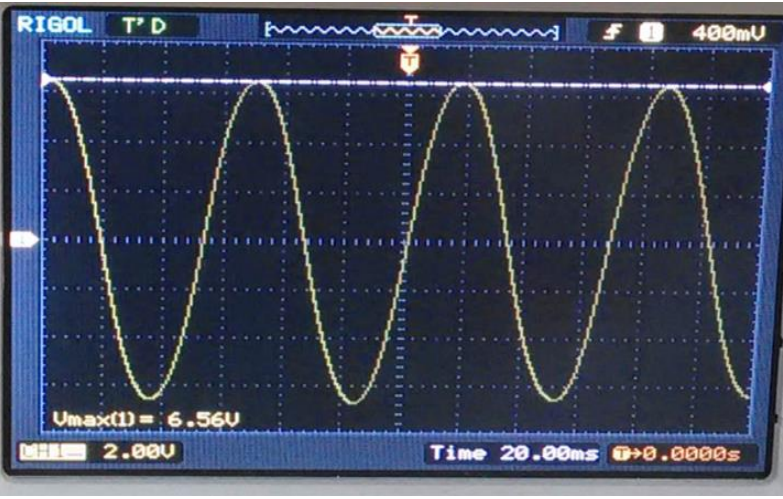

(b) van before filter

Fig. 13. Output waveforms of single phase five-level inverter based on two serie connected CN0196 Hbridge.

After successfully implementation of the ESCS code on Arduino 2560 hardware, the generated PWM switching pulses are connected to five-level DC/AC inverter as it was previously presented in Fig.10. Fig.13 shows the inverter output voltage before and after filtering. The oscilloscope was set to $10 \mathrm{~V} /$ division and 
each DC-link of H-bridge (cell-a1 and a2) was supplied by 36V. So as it is shown on Fig.13a the inverter output voltage contents five levels and the maximum voltage is $7.12 \mathrm{~V}(71.2 \mathrm{~V})$ close to $7.2 \mathrm{~V}$. Also after filtering the output voltage of inverter is similar to the sine with the maximum amplitude equal to $6.56 \mathrm{~V}$ (65.6V) with $50 \mathrm{~Hz}$ as frequency. Thus, the generated code of SV-CBPWM deployed on Arduino 2560 platform shows satisfactory results. Therefore MBD is validated and approved as a way to develop such of control scheme.

\section{Conclusion}

This paper has successfully used model-based design workflow to develop and implement an embedded control software for multilevel converter intended to be used for education and research purpose in West Africa countries where such system are mostly unavailable . Firstly, MBD workflow has been described as a process of modelling, simulation, rapid prototyping, deployment and verification. Secondly, MATLAB/Simulink model of a CB-SVPWM for five-level CHB inverter was developped in continuous time using pure Simulink block-sets. Thirdly, the time continuous model of CB-SVPWM model has been extracted from the previous model and modified in order to develop ESCS code by changing the model from continuous time to discrete time using Simulink model tools such as Simulink code advisor tools, and Simulink embedded coder. Finally, ESCS code have been generated and deployed on Arduino 2560 hardware. The simulated and experimented results presented in this paper prove that MBD is a complete design workflow from model creation to complete implementation enabling possibility to mitigate MP issues. The presented work is a first successful implementation of embedded PWM for multilevel converter using a popular embedded hardware platform Arduino 2560. The result of this work can be applied for education purposes in electrical engineering field mostly West Africa countries. However, for industrial applications the same presented CB-SVPWM model may be modified in order to get suitable model for sophisticated embedded hardware platform such as those commercialized by Texas instruments, Xilinx, Speedgoat etc.

\section{References}

[1] Nilanjan, R., C., Balarko, C., Rajat, M., \& Amirnaser, Y. N. (2014). Multi-terminal Direct-Current Grids (1st ed.). John Wiley \& Sons, IEEE Press.

[2] Rabiul, I., Youguang, G., Rajat, N., \& Jianguo, Z. (2014). Power Converters for Medium Voltages Networks (1st ed.). John Wiley \& Sons, IEEE Press.

[3] Liu, J., Yin, X., Zhang, Z., \& Xion, Q. (2007). Study on theory and key technologies of full digital SPWM Implementation for three-level neutral point clamped inverter. Proceedings of the IEEE. Int. Conf. Communications and Circuits (pp. 1287-1291). Kokura, Japan.

[4] Tae-Yoon, M., Suk-Hyun, S., Jin-Ho, K., Sung-Ho, H., \& Jae, W. (2007, Oct. 17-20). Simulation with consideration characteristics and auto-generated code using matlab/simulink. Proceedings of the Int. Conf. Control, Automation and Systems (pp. 1494-1498). COEX, Seoul, Korea.

[5] Darko, H., \& Karel, J. (2005). Rapid control prototyping using matlab/simulink and a DSP-based motor controller. Int. J. Engng Ed., 21(4), 596-605.

[6] On-target rapid prototyping: Practical approach for bootstrapping production ECU software development. Retrieved from http://www.mathworks.com/tagteam/69755_SAECV11_AIPL.pdf

[7] Model-based design streamlines embedded motor control system development. Analog Devices Technical Article. Retrieved from http://www.analog.com/media/en/technicaldocumentation/technical-articles/Model-Based-Design-Streamlines-Embedded-Motor-ControlSystem-Development.pdf 
[8] Rabiul, I., Youguang, G., Rajat, N., \& Jianguo, Z. (2014). A high-frequency link multilevel cascaded medium-voltage converter for direct grid integration of renewable energy systems. IEEE Trans Power Electron, 29(8), 4167-4182.

[9] H-bridge driver circuit using isolated half-bridge drivers. Analog Devices Technical Article. Retrieved from http://www.analog.com/media/en/reference-design-documentation/referencedesigns/CN0196.pdf

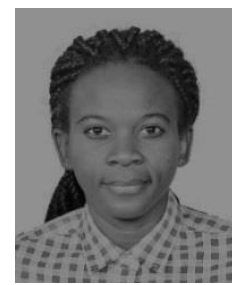

Emilienne Leugoue was born in Douala, Cameroon on February 24, 1990. She received the B.S. and M.S. degrees from University of Ngaoundere, Cameroon in 2011 and 2014 respectively, both in industrial engineering and energetics engineering. Since September 2015 she is enrolled at North China Electric Power University, China. She is currently working toward the Ph.D. degree in field of Electric power system and its automation.

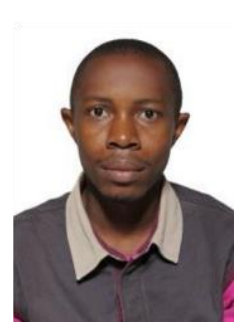

Paul Gistain Ipoum-Ngome was born in Douala in 1989. He received the B.S. and M.S. degrees (2013 and 2015) in electrical engineering from the University of Douala, Cameroon. He also received the DEA in electrical engineering from the same university (2016). Since September 2017, He is working toward the Ph.D. degree in the Department of Electrical Engineering and Automation of Fuzhou University, Fuzhou China. His current research interests include advanced control of motor drives and grid connected systems, harmonics interaction in variable frequency drives (VFDs).

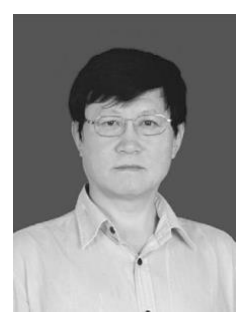

Jianhua Zhang (M'98) was born in Beijing, China, in 1952. He received the B.Sc. and M.Sc. degree in electrical engineering from North China Electric Power University, Baoding, China, in 1982 and 1984, respectively. He was a visiting scholar of the Queen's University, Belfast, U.K., from 1991 to 1992, and was a multimedia engineer of electric power training with CORYS T.E.S.S., France, from 1997 to 1998. Currently, he is working as a professor and the head of the Transmission and Distribution Research Institute, in the School of Electrical and Electronic Engineering of North China Electric Power University, Beijing, China.

He is also a consultant expert of National "973" Plan of the Ministry of Science and Technology. His research interests are in power system security assessment, operation and planning, and microgrid. Prof. Zhang has been an IET fellow since 2005, a member of IEC working group committee for microgrid standards, a member in the PES Committee of China National "973 Project", and also a member of several technical committees.

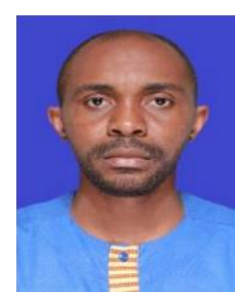

Daniel L. Mon-Nzongo (S'14) was born in Douala, Cameroon, in 1986. He received the B.S. and M.S. degrees in electrical engineering from the University of Douala, Douala, 2010 and 2012, respectively; and the Ph.D. degree in electrical engineering from Fuzhou University, Fuzhou, China, 2018.

Since June 2018, he has been working as postdoctoral researcher with Pearl-Electric Co., in Guangzhou, China. His current research interests include medium-voltage drives, harmonics interaction in large variable-frequency drives, grid-connected systems, power density and efficiency improvements of high-power supplies using Silicon-Carbide (SiC) semiconductors. 